科 学 通 报

\title{
金属酞菁在二氧化钛胶体表面光诱导 电子转移*
}

\author{
王 海 陈德文 ${ }^{* *}$ 徐广智
}

(中国科学院化学研究所分子动态及稳态结构国家重点实验室, 北京 100080)

张先付 许慧君

(中国科学院感光化学研究所, 北京 100101)

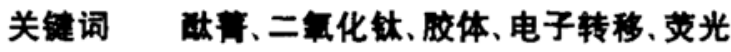

在半导体胶体表面进行的光诱导电子转移过程是当前光化学研究的一个活跃领域. 多数半导体材料导带与价带间的能隙约为 $3 \mathrm{eV}$, 对紫外光有较强的吸收, 但却不能有效利 用日光中的可见光. 若在半导体胶体体系中加人特定的光敏剂, 使之吸附在半导体胶体表 面, 则光敏剂受光激发后, 能够将其电子转移至半导体的导带, 并可进一步传递给其它物质, 使其发生氧化还原反应, 从而扩展了半导体材料的光响应范围 ${ }^{[1]}$.

金属酗青化物在波长为 $600-700 \mathrm{~nm}$ 的可见光范围有强吸收, 且制备简单, 稳定性好, 可望成为新一代人工模拟光合作用的体系. 本文研究了三种水溶性酸菁化物在二氧化钝 $\mathrm{TiO}_{2}$ 胶体表面的光化学与光物理过程, 用 ESR 方法结合紫外 - 可见光谱及荧光光谱研究, 确证了 金属酗青与胶体间发生光诱导电子转移过程, 此为探索有效光能转换体系提供了新的依 据.

\section{1 实 验}

\section{1 材料}

$\mathrm{TiO}_{2}$ 胶体系通过四氮化钩在 $0^{\circ} \mathrm{C}$ 条件下的水解而制得, 并在蒸馏水中进行渗析以纯化胶 体. 当 $\mathrm{pH}>3$ 时, 加人 $2 \%$ 聚乙烯醇作为稳定剂, 最后制得 $\mathrm{pH}=7$ 的清沏透明之胶体溶液. 电子显微镜测之胶体粒度约为 $50-70 \AA$. 水溶性镓钝荎、铝酗青和锌酗菁分别按文献 $[2,3]$ 合 成, 并经三次重结晶提纯.

\section{2 方法}

紫外可见吸收光谱用日立 340 型光谱仪所测. 荧光实验在日立 F-3000 型荧光光谱仪进 行, 激发波长 $610 \mathrm{~nm}$, 涨 $5 \mathrm{~nm}$. 苂光寿命用 HORIBA NAES-1100 型单光子计数仪所 测. ESR 波谱用 Bruker ESP-300 型波谱仪在激光照射的过程中进行检测, 检测前先通 $\mathrm{N}_{2}$ 除 $\mathrm{O}_{2}$.

1993-02-08 收稿, 1993-05-26 收修改稿.

*国家自然科学基金资助项目.

**通讯联系人. 
激光光源为 JD-2 型氦氛激光器, 波长为 $632.8 \mathrm{~nm}$, 输出功率为 $20 \mathrm{~mW}$, 光束直径为 $2 \mathrm{~mm}$. 光源与样品腔的距离为 $50 \mathrm{~cm}$.

\section{2 结 果 与 讨 论}

图 1 所示为铝酞菁的紫外 - 可见吸收光谱, 其中 $679.2 \mathrm{~nm}$ 处之尖峰是铝酗菁单体分子的特 征吸收. 当有 $\mathrm{TiO}_{2}$ 胶体存在时, 该峰值明显降低. 值得注意的是, 最大吸收位置并没有明显 红移,这是由于体系中一部分唒菁分子吸附到 $\mathrm{TiO}_{2}$ 胶体表面的结果. 镓酗菁的紫外可见吸收光谱亦与 此类似.

图 2 是锌酗菁的紫外可见吸收光谱. 可以看 出, 当无胶体存在时, 最大吸收在 $626.0 \mathrm{~nm}$ 处, 这是 锌酗菁二聚体的特征吸收, 即溶液中的锌酗菁分子主 要以二聚体形式存在. 当加人 $\mathrm{TiO}_{2}$ 胶体后,位于 626.0 $\mathrm{nm}$ 处之二聚体特征吸收有所减弱的同时, 又出现 $663.4 \mathrm{~nm}$ 和 $694.0 \mathrm{~nm}$ 两个新的吸收峰 [4]. 此说明溶 液中的二聚体锌酗萻能在胶体表面解离成单体, 并被 吸附于胶体表面上.

上述结果说明溶液中的金属唒菁分子无论以单 体还是以二聚体形式存在, 它们均以单体形式吸附于 $\mathrm{TiO}_{2}$ 胶体表面上.

用 ESR 方法检测结果表明,在无 $\mathrm{TiO}_{2}$ 胶体存在 的金属唒菁体系中, 无论光照时间多长,均无任何 ESR 信号. 但当加人 $\mathrm{TiO}_{2}$ 胶体后, 光照时即很快观察到 金属唒莦在受光激发后, 能将电子转移至半导体的导 带,而本身则变成正离子自由基,如下式所示:

$$
\mathrm{MPc}_{(\mathrm{ad})} \stackrel{\mathrm{h} v}{\longrightarrow} \mathrm{MP}^{*} \mathrm{c}_{(\mathrm{dd})} \stackrel{\mathrm{TiO}_{2}}{\longrightarrow}(\mathrm{MPc}) \div+\mathrm{e}^{-}\left(\mathrm{TiO}_{2} / \mathrm{CB}\right)
$$

由于金属酰羙分子的中心络合原子不同, 因而其正离 子基的 $g$ 值也略有差异, 如表 1 所示. 由此便确切地

表 1 金属酗菁正离子基的 $g$ 值

\begin{tabular}{c|c|c|c}
\hline$(\mathrm{MPc})^{+}$ & 'Al-Pc & Ga-Pc & Zn-Pc \\
\hline$g$ & 2.0034 & 2.0040 & 2.0027 \\
\hline
\end{tabular}

证明了金属酗菁分子与 $\mathrm{TiO}_{2}$ 胶体粒子之间发生了光 诱导电子转移, 其整个过程可见图 3 所表述. 循环伏 安实验结果表明 ${ }^{[7,8]}, \mathrm{TiO}_{2}$ 胶体导带与价带之间的能隙为 $3.0 \mathrm{eV}$, 而基态金属唒菁的氧化电位为 $1.18 \mathrm{eV} / \mathrm{NHE}$,

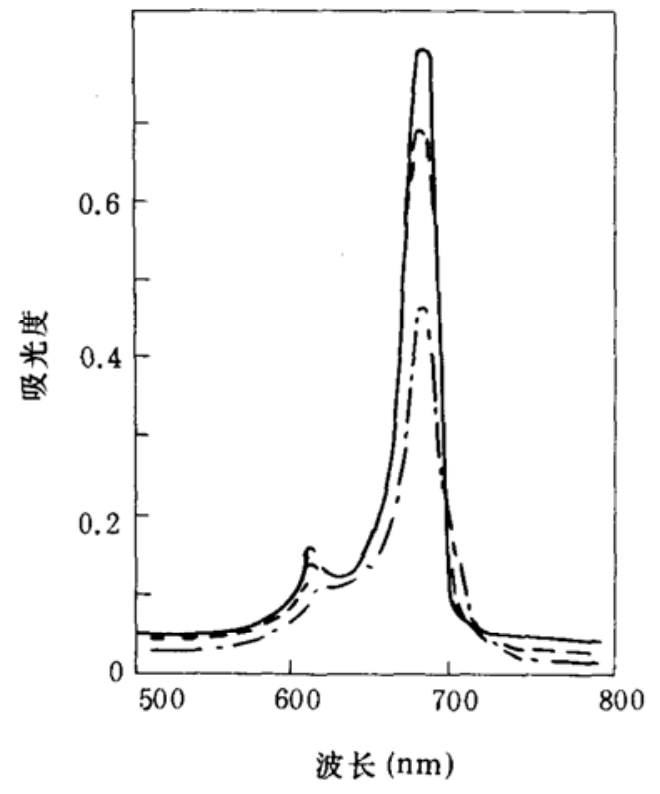

图 1 铝酰蓄的 UV-Vis 吸收光谱 $-\operatorname{AlPc}\left(5 \times 10^{-6} \mathrm{~mol} / \mathrm{L}\right),---\operatorname{AlPc}\left(5 \times 10^{-6}\right.$ $\mathrm{mol} / \mathrm{L})+\mathrm{TiO}_{2}\left(5 \times 10^{-4} \mathrm{~mol} / \mathrm{L}\right),-\cdots \quad-\cdots \mathrm{AlPc}$ $\left(5 \times 10^{-6} \mathrm{~mol} / \mathrm{L}\right)+\mathrm{TiO}_{2}\left(1 \times 10^{-3} \mathrm{~mol} / \mathrm{L}\right)$

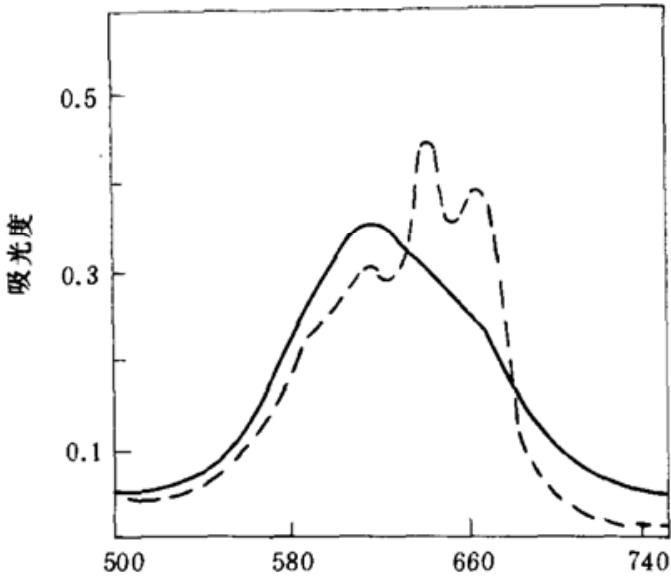

波长 $(\mathrm{nm})$

图 2 锌酸等的 UV-Vis 吸收光谱 $-\mathrm{ZnPc}\left(5 \times 10^{-6} \mathrm{~mol} / \mathrm{L}\right),---\mathrm{ZnPc}$ $\left(5 \times 10^{-6} \mathrm{~mol} / \mathrm{L}\right)+\mathrm{TiO}_{2}\left(5 \times 10^{-4} \mathrm{~mol} / \mathrm{L}\right)$ 


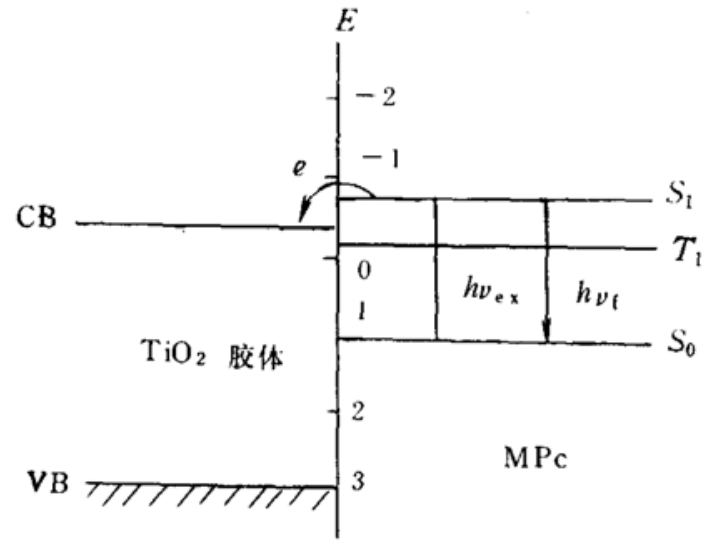

图 3 金属酰菁 $\mathrm{MPc}$ 在 $\mathrm{TiO}_{2}$ 胶体表面光诱导电子 转移过程示意 图中电位值以标准氢电极作参考

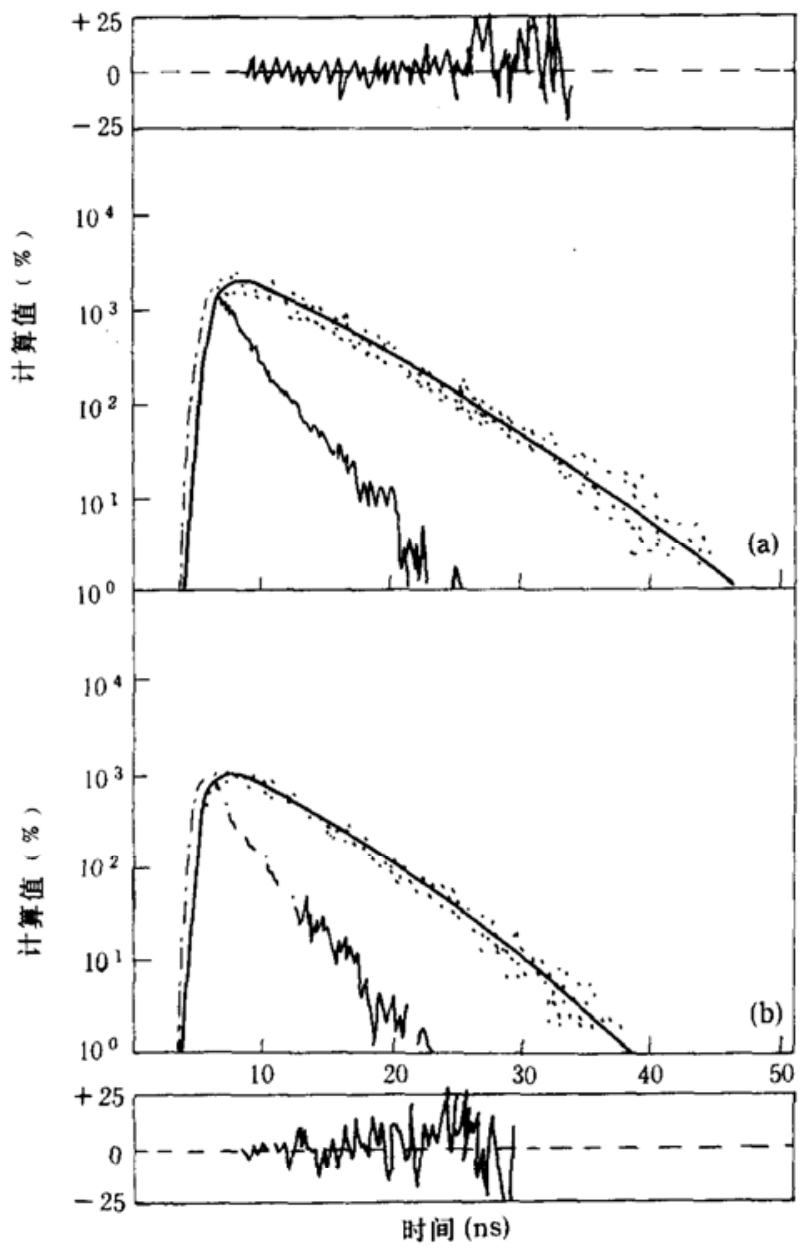

图 4 金属唒蓄的苂光衰减过程 (浓度 $5 \times 10^{-6} \mathrm{~mol} / \mathrm{L}$, 激发波长610nm)

(a) 无 $\mathrm{TiO}_{2}$ 胶体存在, (b)有 $\mathrm{TiO}_{2}$ 胶体存在 $\left(1 \times 10^{-4} \mathrm{~mol} / \mathrm{L}\right)$
其单重 1 态 $\left(S_{1}\right)$ 电位为 $-0.63 \mathrm{eV} / \mathrm{NHE}$, 三重 1 态 $\left(T_{1}\right)$ 电位为 $-0.02 \mathrm{eV} / \mathrm{NHE}$. 从图 3 可以看 出, $S_{1}$ 态的能量高于胶体导带的能量, 而 $T_{1}$ 态的 能量则低于胶体导带的能量. 从热力学角度分 析, 处于基态 $\left(S_{0}\right)$ 的金属酗菁分子在光激发后跃 迁到 $S_{1}$ 态又直接将电子转移到胶体的导带, 而 自身变为正离子基 $\mathrm{MPc}^{+}$是完全可能的. 其过 程可写为:

$$
\begin{aligned}
& \operatorname{MPc}\left(S_{0}\right)+h v_{\text {ex }} \longrightarrow \operatorname{MPc}^{*}\left(S_{1}\right) \\
& \operatorname{MPc}^{*}\left(S_{1}\right) \longrightarrow \operatorname{MPc}\left(S_{0}\right)+h v_{f}
\end{aligned}
$$

$\mathrm{MPc}^{*}\left(S_{1}\right)+\mathrm{TiO}_{2}(\mathrm{CB}) \longrightarrow \mathrm{MPc}^{+}+\mathrm{e}^{-}\left(\mathrm{TiO}_{2} / \mathrm{CB}\right)$ 苂光光谱的研究进一步验证了上述论断. 当用 $610 \mathrm{~nm}$ 的人射光激发时, 随着体系中 $\mathrm{TiO}_{2}$ 胶体浓 度的增加, 铝唒菁的发射光强度不断降低. 当 $\mathrm{TiO}_{2}$ 胶体浓度从零增加到 $5 \times 10^{-4} \mathrm{~mol} / \mathrm{L}$ 时, 铝酗菁的荧光强度降低了约 $70 \%$. 尽管在有 $\mathrm{TiO}_{2}$ 存在时, 铝酗菁在 $610 \mathrm{~nm}$ 处的吸收降 低约近 $10 \%$, 仍能看出胶体粒子对荧光有 很明显的猝灭作用. 镓酗菁和锌酗菁的苂 光光谱也有类似的结果. 为了探求荧光猝 灭机理,我们进一步测量了金属酗菁的苂 光寿命.结果如图 4 所示, 当无胶体存在 时, 镓酗菁的荧光呈单指数式衰减, 其寿命 为 4.69ns. 当加人 $\mathrm{TiO}_{2}$ 胶体以后, 镓酗菁的 苂光衰减曲线有所改变, 并按双指数方式 递减, 苂光寿命分别是 $4.33 \mathrm{~ns}$ 和 $1.55 \mathrm{~ns}$. 这说明一部分酞菁分子仍游离于溶液中, 其荧光寿命与不加胶体时相差不大. 另一 部分酗菁分子吸附于 $\mathrm{TiO}_{2}$ 胶体表面, 受光 激发时发生电子转移, 因此其荧光寿命只 有游离酗菁分子的三分之一. 我们假定菼 光寿命的减少主要是由于电子转移所致, 而且吸附于 $\mathrm{TiO}_{2}$ 胶体表面的酿菁分子之 其它辐射的和非辐射衰域过程的速度与无 $\mathrm{TiO}_{2}$ 胶体存在时的速度相同, 则吸附在胶 体上的酗菁分子的实测荧光寿命 $\tau_{\mathrm{obs}}$ 与其 间电子转移速度常数 $K_{\mathrm{et}}$ 之关系为 ${ }^{[9]}$ 


$$
\frac{1}{\tau_{\mathrm{obs}}}=\frac{1}{\tau}+K_{\mathrm{el}},
$$

式中 $\tau$ 为染料分子在无 $\mathrm{TiO}_{2}$ 胶粒存在时的苂光寿命, 而已知 $\tau_{\mathrm{obs}}=1.55 \mathrm{~ns}$ 和 $\tau=4.69 \mathrm{~ns}$, 则 由上式求出光诱导电子转移速度常数为 $K_{\mathrm{et}}=4.32 \times 10^{8} \mathrm{~s}^{-1}$.

由此, 完全证实了光诱导电子转移过程的发生, 并与 ESR 研究结果相互佐证.

\section{参 考文献}

[1] Kamat, P. V., Fox, M. A., Chem. Phys. Letters, 1983, 102: 379.

[2] 张先付、马金石、蒮秀岩等, 自然科学进展, 1992, (2): 157.

[3] Ali, H., Langlois, R., Wagner, J. R. et al., Photochem. Photobiol., 1988, 47: 713.

[4] Shopova, M., Gantchev, T., J. Photochem. Photobiol., B: Biol., 1990, (6) : 49.

[5] Gantchev, T. G., Kaltchev, M. G., Gotchev, G. P., Int. J. Radiat. Biol., 1991, 60: 597.

[6] Raynor, J. B., Robson, M., Andrew, S. M., J. Chem. Soc. Dalton Trans., 1977, (23) : 2360.

[7] Fan, F. F., Bard, A. J., J. Am. Chem. Soc., 1979, 101: 6139.

[ 8] Darwent, J. R., MoCubbin, I., Phillip, D., J. Chem. Soc. Farad. Trans., 1982, 78(2): 347.

[9] Kamat, P. V., Chauvel, J. P., Fessenden, R. W., J. Phys. Chem., 1986, 90: 1389. 